\title{
Artículos
}

\section{Disposición al ahorro de energía eléctrica en los hogares de México}

\section{Willingness to save electricity in Mexican households}

\author{
Dionicio Morales Ramírez* \\ Elías Alvarado Lagunas** \\ Luis Jonás González Del Ángel***
}

\section{Resumen}

El objetivo del trabajo consiste en identificar la disposición al ahorro de energía eléctrica que presentan los hogares de México. Para ello, se analiza un total de 2815 hogares provenientes de la Encuesta Nacional de los Hogares, así como del Módulo Hogares y Medio Ambiente (2017). Además, se estima un modelo de regresión logística para identificar los determinantes de dicha disposición. Los resultados encontrados señalan que sólo el $50.6 \%$ de los hogares presenta una disposición al ahorro de electricidad, y que las características de la vivienda, el tamaño de la localidad, la ubicación geográfica y

* Universidad Autónoma de Tamaulipas, Facultad de Ingeniería "Arturo Narro Siller". Dirección: Campus Tampico-Madero, 89100, Tampico, Tamaulipas, México. Correo: dionicio.morales@gmail.com ORCID: https://orcid.org/00000002-6859-6547

** Universidad Autónoma de Nuevo León, Facultad de Contaduría Pública y Administración. Dirección: Universidad s/n, Cd. Universidad, 66455, San Nicolás de los Garza, Nuevo León, México. Correo: eliaxalvarado@gmail.com ORCID: https:// orcid.org/0000-0002-2751-7718.

*** Universidad Autónoma de Tamaulipas, Facultad de Ingeniería “Arturo Narro Siller". Correo: ljonas@docentes.uat.edu.mx. ORCID: https://orcid.org/00000002-1773-580X. 
la compra de enseres eléctricos con cierta conciencia ecológica son determinantes para explicarla, en tanto que el precio del servicio no fue relevante. Por lo tanto, las estrategias de ahorro deben de provenir principalmente de programas que estimulen los hábitos de uso.

Palabras clave: consumo de electricidad; consumo sustentable; comportamiento proecológico; regresión logística.

\begin{abstract}
The main goal of this work is to measure the willingness of Mexican households to save electrical power. 2815 households were analyzed from National Household Survey and Households and Environment Module (2017). A logistic regression model was applied in order to identify the determinants of the required disposition. Our results show that only $50.6 \%$ of households have the willingness to save electrical power, and that home characteristics, population size, geographical location and acquisition of electrical appliances with ecological awareness are also determinant factors to describe the disposition, above and beyond the price of the electrical power service. Therefore, saving strategies must come mainly from programs designed to stimulate saving usage habits.
\end{abstract}

Keywords: electricity consumption; sustainable consumption; pro-ecological behavior; logistic regression.

\title{
Introducción
}

La electricidad ha sido en los últimos tiempos la principal fuente de energía empleada en los hogares. Este tipo de energía es usada para el funcionamiento de los enseres eléctricos y electrónicos con el único fin de incrementar el confort y la calidad de vida de gran parte de la población, por lo que su uso se encuentra ligado al estilo de vida y al desarrollo de las sociedades (Vicéns, 2008). Sin embargo, debido a que su generación es principalmente a base de combustibles fósiles (petróleo, carbón y gas), su producción es limitada y altamente contaminante, por lo que es necesario cuidar la manera en cómo se usa a fin de contribuir a la conservación de los recursos, así como combatir 
el cambio climático (Larios, 2014; Dietz, Gardner, Gilligan, Stern y Vandenbergh, 2009).

Los avances tecnológicos alcanzados dentro de la sociedad moderna muestran que los nuevos equipos eléctricos y electrónicos han incrementado su eficiencia en el uso de energía, traduciéndose esto en menores consumos de electricidad por equipo. Aunque, por otro lado, se han disminuido sus costos de producción, lo que ha logrado incrementar el acceso a una mayor cantidad de aparatos eléctricos, de manera tal que el efecto final sobre el consumo de electricidad suele ser mayor, a pesar de la eficiencia de los equipos (Paradoja de Jevons). ${ }^{1}$ Por ello, la innovación tecnológica es una solución parcial para cuidar los recursos. Autores como Cone y Hayes (1985), Boudet, Flora y Armel (2016), Poznaka, Laicane, Blumberga, Blumberga y Rosa (2015), Zhang, Wang y Zhou (2014), Abrahamse y Steg (2009), entre otros, señalan que el comportamiento o los hábitos de uso de la energía pueden contribuir de manera fundamental a moderar su consumo. Asimismo, Saidur, Masjuki y Jamaluddin (2007) consideran que los hogares constituyen un grupo importante para implementar estrategias de conservación.

El ahorro de energía puede provenir, entonces, por una mejora en la eficiencia de los equipos y una reducción en la frecuencia de uso. El ahorro por reducción suele asociarse a los comportamientos de cuidado de la energía, como apagar la luz y desenchufar aparatos, entre otras cosas. A este tipo de prácticas se les denomina como de bajo costo y se asocian a una reducción en los niveles de confort del hogar. En tanto que el ahorro por eficiencia suele relacionarse con la compra de aparatos y con modificaciones estructurales realizadas en la vivienda para hacerla más eficiente en el uso de energía, lo cual implica un mayor costo, aumento en la frecuencia de uso de los enseres, y comúnmente un mejor nivel de confort (Karlin, Davis, Sanguinetti, Gamble, Kirkby y Stokols, 2014).

Los factores que incentivan el ahorro de la energía, y en especial de la eléctrica dentro de los hogares, han sido estudiados am-

${ }^{1}$ Esta paradoja señala que el consumo instantáneo de energía de los equipos disminuye al aumentar su eficiencia, pero incrementa la del uso del modelo, lo que termina provocando un incremento en el consumo total (Jevons, 1865). 
pliamente en diversos países (Henry, Ferraro y Kontoleon, 2019; Van den Broek, Walker y Klöckner, 2019; Poznaka, Laicane, Blumberga, Blumberga y Rosa, 2015; Muljono, 2016; Morales y Luyando, 2016; Laicāne, Blumberga, Rošā y Blumberga, 2014; Wang, Zhang, Yin y Zhang, 2011; Ek y Söderholm, 2010; Abrahamse y Steg, 2009; Sardinou, 2007). En México, a pesar de que el tema de la sustentabilidad energética ha sido incluido dentro la estrategia nacional, y se cuenta con normas oficiales para mejorar el consumo de energía de equipos, así como con programas de apoyo al consumidor para la sustitución de enseres eléctricos, o el programa de educación para el ahorro y uso racional de la energía (Lezama, 2014), poco se ha estudiado sobre la disposición al ahorro que presentan los hogares, a pesar de que el consumo interno de electricidad en este sector es el segundo más alto, con $27.4 \%$ del consumo total, sólo por debajo del sector industrial (Sener, 2018). Además, es el responsable aproximadamente del $21 \%$ de las emisiones de gases de efecto invernadero (Lezama, 2014). Por todo ello, el presente puede considerarse un trabajo exploratorio.

El objetivo consiste en identificar los determinantes de la disposición al ahorro de energía eléctrica dentro de los hogares. Para ello, se estima un modelo de regresión logística binaria empleando una muestra de 2815 hogares provenientes de la Encuesta Nacional de los Hogares aplicada en 2017, con datos del módulo Hogares y Medio Ambiente, ambos aplicados por el Instituto Nacional de Estadística y Geografía (INEGI). El desarrollo de este trabajo se lleva a cabo en cuatro secciones: en la primera se presenta la revisión de literatura sobre el comportamiento del consumo de energía eléctrica residencial; en la segunda se describe la metodología empleada, haciendo énfasis en el modelo y las variables empleadas; en la tercera se presentan los resultados de las estimaciones y su interpretación. Por último, se expone la discusión de los hallazgos y las implicaciones prácticas del estudio.

\section{Antecedentes}

La energía eléctrica ha sido un tema ampliamente estudiado desde la perspectiva económica, esencialmente para explicar los factores 
que determinan su demanda (Vergés, 2009). Para ello, su análisis ha empleado datos por hogar, región o país en series de tiempo o en datos de corte transversal. Dentro de las variables que se han empleado para explicar el consumo de energía eléctrica residencial se identifican algunas como: grado de urbanización, número de individuos que habitan en la vivienda, clima de la región, nivel de estudios e ingresos del jefe del hogar, y precio del servicio, entre otras (Houthakker, 1951; Agostini, Plottier y Saavedra, 2012; Athukorala, Wilson, Managi y Karunarathna, 2019).

En general, los trabajos anteriores tienen en común señalar que los factores que afectan la demanda de energía eléctrica domiciliaria están relacionados principalmente con el incremento del ingreso de los hogares, el precio del servicio y el precio de los bienes energéticos sustitutos, así como con las características propias del hogar. Se encuentran comúnmente elasticidades precio menores a uno, comprobando que la demanda de energía eléctrica es un bien inelástico, por lo que las variaciones en el precio o su tarifa no modifican de manera significativa el consumo de electricidad en los hogares.

Sin embargo, desde el enfoque económico poco se ha señalado sobre el ahorro de la energía, o el impacto de las variables que representen determinadas actitudes o prácticas de cuidado sobre la demanda de electricidad. Ello quizás porque desde esta perspectiva no se suele estudiar las conductas, gustos y preferencias que están detrás de las decisiones de consumo o ahorro; aunque algunos autores, como Athukorala, Wilson, Managi y Karunarathna (2019) y Morales y Luyando (2016), han estimado funciones de demanda de energía eléctrica domiciliaria considerando variables que representen algún tipo de práctica de cuidado.

Desde la óptica de la psicología social, el cuidado del medio ambiente se ha estudiado a través del comportamiento llamado proambiental o proecológico (Shrum, McCarty y Lowrey, 1995; Stern, 2000). Asimismo, Corral (2006) señala que este comportamiento está asociado a las acciones que contribuyen a preservar o reducir el uso de los recursos del entorno. De esta manera, algunos resultados de practicar este tipo de conducta suelen ser la minimización del consumo de productos, la reutilización de desechos como en el reciclaje y el compostaje, la utilización de productos amigables para el 
ambiente, el ahorro de energía eléctrica, combustible, agua, así como la disminución en el uso de vehículos, entre otros.

Los estudios en esta área han intentado explicar los determinantes que motivan a los individuos a cuidar el ambiente, la energía y, recientemente, la electricidad, en donde la teoría del comportamiento planeado de Ajzen (1991) ha sido su base teórica. De manera general, esta teoría señala que el comportamiento está precedido de una intención y que ésta se encuentra determinada por las actitudes, las presiones sociales (normas subjetivas), las experiencias pasadas (efecto residual), así como por la facilidad o dificultad para llevar a cabo dicho comportamiento (control percibido de éste).

A pesar de que se ha elaborado una gran cantidad de trabajos para explicar el consumo de electricidad, los resultados aún continúan siendo ambiguos puesto que no existe consenso sobre los efectos de las variables (Özkan, 2016; Mcloughlin, Duffy y Conlon, 2012; Brounen, Kok y Quigley, 2012; Leahy y Lyons, 2010; Yohanis, Mondol, Wright y Norton, 2008; Bartiaux y Gram-Hanssen, 2005; Cramer, Miller, Craig, Hackett, Dietz, Vine, Levine y Kowalczyk, 1985). Una explicación puede atribuirse a que las situaciones contextuales y metodológicas en donde se llevan a cabo los trabajos suelen ser diferentes entre sí. Así, por ejemplo, Guo, Zhou, Zhang, Lu, Chen y Yang (2018) hacen una revisión de la literatura sobre el consumo de energía eléctrica residencial para identificar los factores y teorías que se han empleado para explicar el consumo, principalmente desde la corriente de la psicología social. Los factores identificados se relacionan con el número de integrantes de la familia, su edad, la existencia de niños dentro de ésta, el nivel de educación, la situación económica, así como el tipo de casa. Señalan que en cuanto a la variable educación y en el caso de tener niños, los resultados son aún ambiguos pues hay autores que encuentran una relación positiva y otros no encuentran nada. En términos de la edad de los residentes, se observa que el consumo de electricidad se va incrementando con los años, aunque llega un momento en el que decae, comúnmente después de los 64 años. Respecto al nivel de ingresos y al estatus socioeconómico, se suelen encontrar efectos positivos sobre el consumo; sin embargo, también existe la idea de que un mayor nivel de ingresos le da a las familias acceso a enseres eléctricos más eficien- 
tes, por lo que en un momento dado se pudieran obtener efectos de reducción en el consumo.

Otro trabajo interesante es el desarrollado por Henry, Ferraro y Kontoleon (2019), quienes llevaron a cabo un experimento en aproximadamente 9000 hogares de Estados Unidos, el cual consistió en mandar vía electrónica un reporte del consumo de energía del hogar a un grupo de tratamiento, mientras continuaban con los reportes tradicionales vía correo postal al grupo de control. Sus resultados señalan que en ambos grupos el consumo se redujo aproximadamente $2.9 \%$, por lo que concluyen que los reportes transmitidos electrónicamente son igual de efectivos, aunque más baratos que los enviados por correo postal.

En Europa occidental, Van den Broek, Walker y Klöckner (2019) estudiaron el comportamiento de ahorro en 247 hogares mediante una encuesta y la técnica de ecuaciones estructurales. Sus hallazgos evidencian que, para incentivar la conducta de ahorro, los hacedores de política deben de instaurar programas que estimulen el cambio en los hábitos, así como crear entornos que faciliten el cuidado ambiental.

Otro estudio que emplea encuestas y ecuaciones estructurales es el de Muljono (2016), en Indonesia. Este autor analiza si la exposición a las campañas y los programas para concientizar sobre el uso de la energía en hogares tiene algún efecto sobre el comportamiento de ahorro de electricidad. Sus resultados muestran que la información presentada en los medios de comunicación no tiene un efecto directo sobre la conducta de ahorro, pero sí lo tiene sobre la comunicación interpersonal, la cual a su vez influye en las actitudes ecológicas. Y tanto la comunicación interpersonal como la práctica de actitudes ecológicas tienen efectos directos sobre la conducta de ahorro. En otras palabras, los medios de comunicación no generan un comportamiento directo sobre la conducta, pero sí lo hacen de manera indirecta. Esto último sucede cuando los residentes comparten entre ellos los conocimientos y las habilidades para obtener comportamientos de ahorro.

En Letonia, Laicāne, Blumberga, Rošā y Blumberga (2014) y Poznaka, Laicane, Blumberga, Blumberga y Rosa (2015) analizan el consumo de electricidad dentro de los hogares. En 2014, los prime- 
ros autores llevaron a cabo un estudio en el que dividieron la muestra en un grupo de control y otro de experimentación. A este último le implementaron un medidor inteligente que les mandaba a los usuarios reportes de su consumo en tiempo real. Los resultados indican que los hogares expuestos a tal medidor redujeron en $23 \%$ su consumo, y los que no lo tenían, lo hicieron en 5\%. Además, mencionan que los mayores ahorros se dieron en los hogares que instalaron colectores solares; en cambio, poseer un clima central y televisiones LED produjo que los hogares obtuvieran un menor ahorro de energía. Un año más tarde, Poznaka, Laicane, Blumberga, Blumberga y Rosa (2015) también analizaron el consumo de los hogares mediante la aplicación de encuestas y entrevistas, concluyendo que las principales motivaciones que influyen positivamente en la conducta de cuidado de energía se deben a las expectativas de ahorro de dinero que esperan tener, así como a la información en tiempo real que reciben mediante el medidor inteligente.

En México, Morales y Luyando (2016) han estudiado la relación entre las actitudes y el conocimiento sobre el consumo de energía eléctrica en 370 hogares dentro del Área Metropolitana de Monterrey, Nuevo León. Para ello, emplearon una encuesta semiestructurada y elaboraron un análisis de regresión múltiple empleando datos agregados por hogar. Sus resultados señalan que poseer actitudes de ahorro o buenas prácticas son determinantes para reducir el consumo de electricidad en los hogares, lo que reduce en aproximadamente $13 \%$ el consumo del hogar, cifra muy parecida a la registrada en el trabajo de Ouyang y Hokao (2009).

Ek y Söderholm (2010) aplicaron una encuesta a 1200 hogares de Suecia, y empleando un modelo probit ordenado comprobaron que brindar información específica sobre las medidas de ahorro es más efectivo para estimular la disposición al ahorro que brindar información general. Asimismo, encontraron que las actitudes ambientales tienen un efecto positivo sobre la disposición, en tanto que las variables socioeconómicas, como la educación, la edad, el ingreso y el género, no fueron determinantes de la disposición al ahorro de electricidad.

En la ciudad de Pekín, Wang, Zhang, Yin y Zhang (2011) estudiaron las oportunidades de ahorro en el consumo de energía eléctri- 
ca mediante una encuesta aplicada a 816 residentes del área urbana $\mathrm{y}$, mediante un análisis de regresión logística binaria, encontraron que los beneficios económicos percibidos por ahorrar, las normas sociales, las experiencias pasadas y la edad, son determinantes para incentivar positivamente la disposición al ahorro. En tanto que las disconformidades en el servicio y el tamaño del área de la casa suelen tener impactos negativos sobre la disposición. Respecto a la conciencia ambiental, estos autores no hallaron un efecto sobre la disposición al ahorro.

En 2009 se publicó el trabajo de Abrahamse y Steg (2009), quienes llevaron a cabo un experimento en 314 hogares de la ciudad de Groningen, Holanda. Dichos hogares fueron separados en un grupo de control y otro experimental. Al grupo experimental se le estuvo mandando información durante cinco meses sobre cómo podrían reducir el uso de energía (gas, electricidad y combustible) mediante cambios en su manera de utilizarla. Encontraron que los hogares expuestos a la intervención ahorraron $5.1 \%$ en su consumo de energía, en tanto que el grupo de control lo incrementó en $0.7 \%$. Posteriormente, estimaron una regresión jerárquica para identificar los determinantes del consumo y el ahorro. Sus resultados evidencian que las variables sociodemográficas suelen impactar el uso de energía, en tanto que las psicológicas sólo lo hacen sobre el ahorro de ésta.

En Grecia, Sardinou (2007) estudió la disposición a la conservación de la energía eléctrica dentro de los hogares. Esta autora encontró que la edad tiene un impacto negativo sobre dicha disposición; en otras palabras, conforme aumenta la edad, el número reportado de acciones de conservación tiende a disminuir. En tanto que el género, el nivel de estudios, el tamaño de la casa y el número de cuartos no fueron relevantes para explicar tal disposición. Además, y contrario a lo esperado, encontró que conforme crece el número de integrantes del hogar, también se incrementa la disposición o el número de prácticas de ahorro (esto también se reportó en los trabajos de Van Raaij y Verhallen, 1983). En cuestiones de ingreso, halló también una relación positiva; es decir, las personas con mayores ingresos son quienes cuentan con más disposición al ahorro. Ello se debe posiblemente a que tienen acceso a créditos que les permiten invertir en equipos e infraestructura para alcanzar mejoras energéticas den- 
tro del hogar. Por último, señala que poseer información sobre los problemas ambientales y estar consciente de cómo nuestras acciones contribuyen a incrementar dichos problemas, tiende a incrementar positivamente la disposición a la conservación, por lo que resulta importante estimular la conciencia ambiental para el cuidado de la electricidad.

\section{Metodología}

\section{La muestra y los datos}

Se emplea información extraída de la Encuesta Nacional de los Hogares (ENH) y del Módulo de Hogares y Medio Ambiente (Mohoma), aplicados en 2017 por el INEGI. La ENH brinda información sobre las características de las viviendas y datos sociodemográficos acerca de los integrantes del hogar. La encuesta del Mohoma capta información estadística sobre las actitudes, prácticas o acciones que se realizan dentro de los hogares sobre el uso de los recursos naturales o su degradación. Ambas encuestas tienen como unidad de selección la vivienda, y la de observación es el hogar; además generan resultados a nivel nacional (INEGI, 2018a; 2018b).

Para aprovechar la información de ambas encuestas, primero se tuvo que hacer una unión de las variables mediante un folio, el cual sirvió para identificar la misma vivienda en las dos bases de datos. Al hacer esta fusión, el total de viviendas que registra Mohoma se redujo de 14505 a 14279 . Asimismo, se optó por trabajar sólo con los hogares que poseen recibo de energía eléctrica y que presentan un consumo mayor o igual a 25 kilowatts por mes, el cual es el mínimo cobrado por la Comisión Federal de Electricidad (CFE). También se excluyeron los hogares que estuvieran registrados con tarifa de alto consumo (DAC). Por último, se calculó el logaritmo natural de los kilowatts consumidos al mes y se construyó una gráfica de box plot para identificar datos atípicos en la muestra, los cuales fueron eliminados. Después de todo este procedimiento, la muestra con la que se trabajó fue de 2815 viviendas. 


\section{Disposición al ahorro en los hogares (DA)}

La construcción de esta variable se hace desde la perspectiva de la psicología social, en concordancia con la Teoría del Comportamiento Planeado de Ajzen (1991), en donde se supone que una conducta específica, como la de ahorro de energía, es el reflejo de un conjunto de actitudes, creencias, normas sociales y contexto en donde se desarrollan las personas. En nuestro caso consideramos seis preguntas actitudinales de tipo dicotómico, las cuales indagan sobre prácticas de ahorro, como apagar las luces cuando no se emplean, desconectar aparatos, planchar mayor cantidad de ropa en un solo momento, desconectar cargadores de celular y laptop cuando no se usan, así como revisar las instalaciones eléctricas durante la última semana. Lo anterior puede expresarse de la siguiente forma:

$$
D=\frac{1}{n} \sum_{i=1}^{6} P_{i}
$$

El resultado obtenido en la ecuación [1] toma valores entre 0 y 1 , por lo que fue necesario redondear bajo el siguiente criterio: si D $>.5$, entonces $\mathrm{DA}=1 ; \mathrm{y}$ si $\mathrm{D} \leq .5$, entonces $\mathrm{DA}=0$. Esto implica que para clasificar a un hogar como poseedor de una conducta de disposición al ahorro ( $\mathrm{DA}=1)$, éste debe llevar a cabo al menos cuatro de seis actitudes o prácticas de ahorro. Caso contrario, si el hogar presenta tres o menos actitudes, se clasifica como sin disposición $(\mathrm{DA}=0)$.

\section{El modelo}

Se desarrolla un modelo econométrico para identificar las variables que determinan la disposición al ahorro en los hogares de México. Debido a que la variable dependiente DA toma el valor de 0 y 1 , es posible emplear dos tipos de modelos: el logit y el probit. En nuestro caso se emplea el logit, al igual que el trabajo de Wang, Zhang, Yin y Zhang (2011), quienes señalan que este tipo de regresión es preferido para modelar las decisiones de maximización de la utilidad. Y ya 
que la decisión de ahorro depende de la utilidad esperada por dicho comportamiento, el modelo de logit es una mejor opción respecto al probit (Börsch-Supan, 1990). El modelo se especifica de la siguiente manera:

$$
\operatorname{Logit}(R)=\frac{1}{1+e^{-D}} \quad D=\beta_{0}+\sum_{i=1}^{n} \beta_{i} x_{i}+\varepsilon_{i}
$$

en donde $\mathrm{R}$ es la variable discreta codificada que hace referencia a la disposición al ahorro (DA), en tanto que $\mathrm{D}$ es la variable latente continua con la que se construye DA. Los coeficientes de $\beta$ corresponden al vector de parámetros a ser estimados mediante máxima verosimilitud. En tanto que el termino de error es captado por $\varepsilon$, el cual se asume con distribución normal estándar.

De acuerdo con la literatura revisada, se emplean las siguientes variables referentes a las características de la vivienda: número de cuartos, número de integrantes del hogar, número de focos, y posesión de enseres eléctricos, como refrigerador, televisión, lavadora, ventilador, plancha, computadora personal y aire acondicionado (en donde $1=$ sí tiene, y $0=$ no tiene). Además, se incluye una pregunta de tipo conductual sobre la preferencia de compra de los aparatos eléctricos, la cual cuenta con las categorías: 1) compra por eficiencia energética, 2) compra por etiqueta ecológica, 3) compra por la marca, 4) compra por el precio, 5) no ha comprado. El precio promedio del servicio de energía eléctrica también es incluido para verificar si las cuestiones económicas al respecto tienen un efecto sobre la disposición al ahorro. Por último, para identificar las diferencias atribuidas a las características de cada lugar, como el clima o las cuestiones culturales, se incluyó la variable región, integrada por las categorías: 1) centro norte, 2) centro sur, 3) noreste, 4) noroeste, 5) occidente, 6) oriente, 7) sureste y 8) suroeste. También se incorporó el tamaño de la localidad, el cual está integrado por cuatro categorías: a) 100000 y más habitantes, b) 15000 a 99999 habitantes, c) 2500 a 14999 habitantes, y d) menos de 2500 habitantes. 


\section{Resultados}

Las estadísticas básicas señalan que de las 2815 viviendas que integran la muestra del análisis, $96.6 \%$ de ellas son del tipo casa independiente, $2.9 \%$ son condominios y $0.4 \%$ son viviendas en vecindad. El $86.8 \%$ tiene un patio, $0.1 \%$ cuenta con paneles solares, y tienen en promedio 21 años de antigüedad. El 50\% de los hogares pertenece a un estrato socioeconómico medio bajo, $24.6 \%$ al bajo, $19.3 \%$ al medio alto y sólo $6.1 \%$ al alto. Además, un hogar en promedio está integrado por cuatro personas, que habitan una vivienda con cuatro cuartos y con aproximadamente siete focos.

En cuestiones del gasto de energía eléctrica, se encontró que 94.5\% de los hogares hace pagos de manera bimestral y el resto lo hace de forma mensual. En el Cuadro 1 se presenta el pago por el servicio de energía eléctrica, el consumo en kilowatts y el precio promedio separado por tipo de localidad. Nótese que las localidades más grandes son las que pagan y consumen más energía eléctrica por hogar. De acuerdo al INEGI (2010), ${ }^{2}$ las áreas compuestas por localidades con más de 2500 habitantes suelen clasificarse como urbanas, y aquellas con cifras menores a ésta suelen denominarse rurales.

\section{Cuadro 1}

Pago y consumo bimestral de electricidad en viviendas

\begin{tabular}{lcccc}
\hline & \multicolumn{4}{c}{ Tamaño de la localidad } \\
\cline { 2 - 5 } \multicolumn{1}{c}{ Variable } & $\begin{array}{c}\mathbf{1 0 0 0 0 0} \text { y más } \\
\text { habs. }\end{array}$ & $\begin{array}{c}\mathbf{1 5 0 0 0} \boldsymbol{\mathbf { 9 9 }} \\
\mathbf{9 9 9 9} \text { habs. }\end{array}$ & $\mathbf{2 5 0 0} \boldsymbol{\mathbf { 1 4 9 9 9 } \text { habs. }}$ & $\begin{array}{c}\text { Menos } \\
\text { 2500 habs. }\end{array}$ \\
\hline Pago & 486.52 & 312.56 & 277.09 & 238.73 \\
Consumo (kW) & 310.54 & 252.4 & 230.41 & 205.42 \\
Precio promedio & 1.45 & 1.17 & 1.12 & 1.12 \\
Viviendas & 979 & 474 & 453 & 909 \\
\hline
\end{tabular}

Fuente: Elaboración propia.

${ }^{2}$ Véase la sección Cuéntame, en la página del INEGI. http://cuentame.inegi. org.mx/poblacion/rur_urb.aspx?tema_P 
En el Cuadro 2 se aprecian las variables actitudinales que se tomaron en cuenta para la estimación de la DA. Asimismo, se encontró de manera general que $50.6 \%$ de los hogares sí muestran disposición al ahorro.

\section{Cuadro 2}

Distribución de frecuencias porcentuales de las preguntas que integran la disposición al ahorro (DA)

Durante la última semana, ¿realizaron algunas

de las siguientes prácticas para ahorrar energía en este hogar?

Sí (\%)

1. Apagar las luces

98.4

2. Desconectar aparatos

79.6

3. Planchar en mayores cantidades

4. Desconectar cargadores

5. Desconectar laptop

6. Revisión de instalaciones eléctricas

Fuente: Elaboración propia.

En el Cuadro 3 se presenta la distribución porcentual de la clasificación obtenida en la variable DA por hogar, controlando por el tamaño de la localidad. Se encuentra que las áreas urbanizadas tienden a presentar mayor disposición al ahorro dentro del hogar.

\section{Cuadro 3}

Porcentaje de los hogares con y sin disposición al ahorro

\begin{tabular}{lcccc}
\hline & \multicolumn{4}{c}{ Tamaño de la localidad } \\
\cline { 2 - 5 } $\begin{array}{l}\text { Disposición } \\
\text { al ahorro }\end{array}$ & $\begin{array}{c}\mathbf{1 0 0 0 0 0} \text { y más } \\
\text { habs. }\end{array}$ & $\begin{array}{c}\mathbf{1 5 0 0 0} \boldsymbol{a} \\
\mathbf{9 9 9 9 9} \text { habs. }\end{array}$ & $\begin{array}{c}\text { 2500 a 14999 } \\
\text { habs. }\end{array}$ & $\begin{array}{c}\text { Menos de } \\
\text { 2500 habs. }\end{array}$ \\
\hline Sí =1 & 61.7 & 54.0 & 51.2 & 36.6 \\
No $=0$ & 38.3 & 46.0 & 48.8 & 63.4 \\
Viviendas & 979 & 474 & 453 & 909 \\
\hline
\end{tabular}

Fuente: Elaboración propia.

Uno esperaría que los hogares que se clasifican con disposición al ahorro realicen un consumo de energía eléctrica más bajo, por lo 
que en el Cuadro 4 se presenta el consumo per cápita promedio medido en kilowatts. La comparación se hace en términos per cápita para tratar de controlar las variaciones en los hogares atribuidas al número de sus integrantes.

\section{Cuadro 4}

Disposición al ahorro y consumo en kilowatts

\begin{tabular}{|c|c|c|c|c|}
\hline \multirow[b]{2}{*}{$\begin{array}{l}\text { Disposición } \\
\text { al ahorro }\end{array}$} & \multicolumn{4}{|c|}{ Consumo per cápita en kilowatts } \\
\hline & $\begin{array}{c}100000 \text { y más } \\
\text { habs. }\end{array}$ & $\begin{array}{c}15000 a \\
99999 \text { habs. }\end{array}$ & $\begin{array}{c}2500 \text { a } 14999 \\
\text { habs. }\end{array}$ & $\begin{array}{l}\text { Menos de } \\
2500 \text { habs. }\end{array}$ \\
\hline Sí = 1 & 105.9 & 82.8 & 66.6 & 62.1 \\
\hline No $=0$ & 121.5 & 92.6 & 78.4 & 66.0 \\
\hline Viviendas & 979 & 474 & 453 & 909 \\
\hline
\end{tabular}

Fuente: Elaboración propia.

En el Cuadro 4 se puede observar también que existen diferencias visibles entre los hogares que poseen disposición al ahorro y los que no, sobre todo en las áreas urbanas (con más de 2500 habitantes). Caso contrario ocurre en el área rural (con menos de 2500 habitantes), en donde el consumo per cápita de electricidad es muy parecido.

El inventario de equipos con los que cuenta una vivienda en México es de aproximadamente diez enseres eléctricos, los cuales en promedio tienen una antigüedad de cuatro años, y de todos, el refrigerador es el más antiguo, habiendo sido adquirido aproximadamente en 2010 (Cuadro 5).

En términos de la compra de bienes considerando ciertos atributos, se encontró que $41.9 \%$ de los hogares adquirió sus más recientes aparatos eléctricos por cuestiones económicas (precio); $16.1 \%$ consideró la marca del producto; $15.2 \%$ valoró la eficiencia energética; $2.2 \%$ tomó en consideración que tuviera etiqueta ecológica; y el resto no había comprado. 


\section{Cuadro 5}

Enseres eléctricos: cantidad y antigüedad promedio

\begin{tabular}{lcc}
\hline Equipos & Cantidad & Años \\
\hline Refrigerador & 1.0 & 6.8 \\
Televisor o pantalla plana & 1.5 & 3.4 \\
Lavadora & 1.0 & 5.5 \\
Ventilador eléctrico (abanico) & 1.9 & 3.5 \\
Computadora (PC de escritorio) & 1.0 & 5.1 \\
Equipo de aire acondicionado mini split & 1.6 & 3.9 \\
Computadora (laptop) & 1.2 & 3.0 \\
Plancha & 1.0 & 4.7 \\
\hline
\end{tabular}

Fuente: Elaboración propia.

\section{Análisis de impacto}

Los resultados de la estimación de la ecuación [2] permiten identificar el impacto de las variables propuestas sobre la DA (Cuadro 6). El modelo permite clasificar correctamente el $67.6 \%$ de los casos de forma general. Respecto a la sensitividad, es decir, la probabilidad de clasificar adecuadamente los casos con disposición al ahorro, ésta es de $70.2 \%$; mientras que la especificidad, es decir, la probabilidad de clasificar adecuadamente los casos sin disposición al ahorro, es de $64.9 \%$.

Para identificar la bondad del ajuste del modelo se empleó el $\mathrm{R}$ cuadrado de Nagelkerke, el cual indica que las variables consideradas en el modelo explican el $22.0 \%$ de la variabilidad. Y para verificar que dicho ajuste del modelo es estadísticamente significativo, se estimó el estadístico de Hosmer-Lemeshow $(\mathrm{Cg})(\mathrm{p}$-valor $=0.674)$. Ambas pruebas señalan que los resultados de la estimación son adecuados, por lo que se puede proceder a la interpretación de los coeficientes estimados (odds ratio). 


\section{Cuadro 6}

Estimaciones del modelo

\begin{tabular}{|c|c|c|c|}
\hline Variables & & $\begin{array}{c}\text { Coeficiente } \\
\text { (B) }\end{array}$ & $\begin{array}{l}\text { Odds ratio } \\
\exp (B)\end{array}$ \\
\hline Precio promedio del servicio & & 0.03 & 1.030 \\
\hline \multirow{4}{*}{$\begin{array}{l}\text { Compra de aparatos eléctricos } \\
\text { considerando: }\end{array}$} & Eficiencia energética & 0.563 & $1.757^{*}$ \\
\hline & Etiqueta ecológica & 0.815 & $2.260^{*}$ \\
\hline & Marca & 0.339 & $1.403 *$ \\
\hline & Precio & 0.121 & 1.128 \\
\hline \multirow{10}{*}{$\begin{array}{l}\text { Enseres eléctricos } \\
\text { con los que cuenta }\end{array}$} & Refrigerador & 0.122 & 1.130 \\
\hline & Televisión & 0.255 & 1.291 \\
\hline & Lavadora & 0.394 & $1.482 *$ \\
\hline & Ventilador & 0.006 & 1.006 \\
\hline & Plancha & 0.802 & $2.231 *$ \\
\hline & Computadora & 0.633 & $1.883^{*}$ \\
\hline & Regadera eléctrica & 0.324 & 1.382 \\
\hline & Aire acondicionado & 0.101 & 1.106 \\
\hline & Calentador gas & 0.375 & $1.455^{*}$ \\
\hline & Calentador solar & 0.284 & 1.329 \\
\hline \multirow[t]{3}{*}{ Características de la vivienda } & Cuartos & 0.000 & 1.000 \\
\hline & Focos & 0.049 & $1.050 *$ \\
\hline & Integrantes del hogar & 0.138 & $1.148^{*}$ \\
\hline \multirow[t]{7}{*}{ Región } & Centro norte & 0.509 & $1.664^{*}$ \\
\hline & Centro sur & 0.546 & $1.726^{*}$ \\
\hline & Noreste & 0.924 & $2.52 *$ \\
\hline & Occidente & 0.706 & $2.025^{*}$ \\
\hline & Oriente & 0.580 & $1.786^{*}$ \\
\hline & Sureste & 0.627 & $1.872 *$ \\
\hline & Suroeste & 0.45 & $1.569^{*}$ \\
\hline \multirow[t]{3}{*}{ Tamaño localidad } & 100000 y más habs. & 0.544 & $1.723 *$ \\
\hline & 15000 a 99999 habs. & 0.352 & $1.422 *$ \\
\hline & 2500 a 14999 habs. & 0.357 & $1.429 *$ \\
\hline Constante & & -3.457 & $0.032 *$ \\
\hline
\end{tabular}

Fuente: Elaboración propia. 


\section{Discusión}

Las estimaciones del modelo propuesto señalan que las variables presentan un efecto sobre la disposición al ahorro de electricidad dentro del hogar. De manera específica, se observa que la compra de equipos electrónicos con etiqueta ecológica incrementa 2.2 veces más la DA respecto de las personas que no han comprado un equipo recientemente; sucede de la misma forma para los que compran considerando la eficiencia energética (1.7) y la marca (1.3). Estos resultados son similares a los presentados por autores como Sardinou (2007), Morales y Luyando (2016), quienes señalan que el conocimiento y las actitudes para favorecer el cuidado del medio ambiente son variables efectivas para reducir el consumo. En otras palabras, presentar una conciencia proecológica contribuye a incrementar la disposición al ahorro.

El precio promedio del servicio de energía eléctrica no fue estadísticamente significativo o diferente de cero. Por lo tanto, una estrategia basada en precios no parece tener un efecto certero para estimular el ahorro dentro de los hogares. Aunque es importante recordar que el sector eléctrico mexicano está regulado por el gobierno, quien es el que establece los precios y brinda subsidios; por ello, el precio en este sector pudiera no captar de manera adecuada las señales del mercado. Respecto a lo anterior, Athukorala, Wilson, Managi y Karunarathna (2019) argumentan que en los mercados regulados en donde se otorgan subsidios y existen estructuras tarifarias de bloque, el efecto del subsidio suele anular el efecto racionador del precio, lo cual parece reflejarse en nuestros resultados.

Respecto al inventario de equipos eléctricos dentro del hogar, se puede observar que, de los diez enseres propuestos, sólo cuatro presentaron un efecto positivo sobre la DA. Así, por ejemplo, tener plancha incrementa la disposición al ahorro 2.2 veces más respecto de quienes no la tienen, al igual que tener computadora, lavadora y calentador de gas. Ello sugiere que los hogares que poseen este tipo de equipamiento ponen especial atención en su uso. El resto de los enseres eléctricos no hace una diferencia en la disposición, posiblemente porque, como se observa en el Cuadro 5 , son equipos relativamente nuevos y por consiguiente cumplen con las especificaciones normativas sobre el consumo eficiente. 
El número de focos y el total de integrantes del hogar son también variables relevantes. En el caso de los primeros, se encontró que el aumento de un foco dentro de la vivienda incrementa 1.0 veces más la DA, lo que implica que mientras más focos tenga la casa, existe mayor disposición para cuidar cuándo se prenden y cuándo se apagan. Respecto al total de integrantes del hogar, se observa también un efecto positivo sobre la DA; en otras palabras, un miembro nuevo tiende a incrementar 1.1 veces la disposición al ahorro. Estos resultados son coherentes con los encontrados por Sardinou (2007), y también por Van Raaij y Verahllen (1983).

El tamaño de la vivienda fue aproximado por el número de cuartos, el cual no fue significativo. Contrario a los resultados de Wang, Zhang, Yin y Zhang (2011), quienes señalan que mientras más grande sea el terreno de la casa, menor será la disposición al ahorro; es decir, mientras más grande sea el lugar, más demanda de electricidad se generará porque la gente tiene menos disposición a cuidarla. Sin embargo, al igual que el trabajo de Sardinou (2007), nuestros resultados revelan que los hogares que poseen enseres eléctricos tienden a presentar mayor cantidad de acciones de cuidado, independientemente del tamaño de la vivienda.

La región geográfica y el tamaño de la localidad fueron variables que se emplearon para capturar, entre otras cosas, los efectos referentes al grado de urbanismo, las condiciones climatológicas o las cuestiones culturales. Los resultados indican que existe heterogeneidad en la DA de acuerdo al tamaño de la localidad y la región. Específicamente, encontramos que los hogares ubicados en las localidades con mayor cantidad de habitantes o más urbanizadas, tienden a presentar 1.7 veces más disposición al ahorro respecto aquellas denominadas rurales. Asimismo, se comprobó que la disposición al ahorro depende de la zona geográfica en donde se encuentren ubicados los hogares, siendo los del noreste (2.5) y occidente (2.0) los que mayor disposición presentan respecto a los del noroeste. Dichas diferencias pueden ser explicadas por las características físicas y climatológicas de cada lugar, aunque también son el reflejo de los usos y costumbres arraigados en el sistema de valores y creencias que definen el estilo de vida de cada lugar.

De acuerdo con Inglehart (1997), las actitudes, valores y creencias que definen una intención de comportamiento dentro la so- 
ciedad, dependen de la etapa de desarrollo o momento histórico e institucional en el que ésta se encuentre. Para que dicha intención se convierta en una conducta efectiva, se requiere de un contexto social, ambiental y económico, en donde se generen oportunidades para que las personas lleven a cabo dicha conducta; y de motivación, con objeto de que la mantengan a través del tiempo.

En este sentido, Lezama (2014) nos indica que los esfuerzos que se han hecho en México en cuestiones de normatividad para buscar la eficiencia de los enseres eléctricos, así como los diversos programas y talleres educativos que se llevan a cabo dentro del sector energético del país, son pertinentes y están tratando de generar ese contexto para que las personas cuiden la energía. Aunque reconoce que la normatividad mexicana contribuye de manera parcial a la crisis energética y al cambio climático, ya que por un lado promueve el ahorro o consumo moderado, y por otro, el sistema económico estimula el desarrollo de nuevos equipos, los cuales no se encuentran incluidos dentro de las normas de eficiencia energética, incumpliendo con ello el objetivo de bajo consumo. Peor aún, el denominado modo "reposo" en ciertos aparatos, como los celulares o computadoras, para evitar que sean desconectados, hace que los esfuerzos de cuidado de la energía y del medio ambiente a través de las normas sean poco efectivos.

Por último, el modelo propuesto presenta medidas de ajuste aceptables pero bajas, lo cual sugiere seguir explorando el tema con nuevas variables explicativas, así como incluir preguntas que capten de manera específica una conducta proecológica (Corral, 2010).

\section{Conclusiones}

En este trabajo exploratorio se estudian los determinantes de la disposición al ahorro en una muestra de hogares de México. Para ello se construyó una variable que capta dicha disposición (DA) y después se estimó un modelo de regresión logística con datos provenientes de la Encuesta Nacional de los Hogares (ENH) y del Módulo de Hogares y Medio Ambiente (Mohoma), aplicados en 2017 por el INEGI. 
Los hallazgos del análisis empírico demuestran que 50.6\% de los hogares en México presentan una disposición al ahorro, lo que representa una oportunidad para estimular la adopción de prácticas que incentiven el cuidado del recurso dentro de los hogares. Para ello, se debe fomentar un cambio en los hábitos de uso o, como lo plantea Inglehart (1997), transitar de un proceso de industrialización (modernidad) basado en pensamientos individuales y materialistas, a uno de posmodernidad, cimentado en pensamientos de bienestar y desmaterialización, lo cual implica un cambio profundo en el sistema de valores y creencias de la sociedad. Además, se debe continuar apoyando los programas para la sustitución de enseres eléctricos viejos por nuevos, así como brindar incentivos para el desarrollo de viviendas térmicas que sean capaces de moderar el consumo de energía y, con ello, contribuir al cuidado de los recursos y del medio ambiente de manera integral.

Además, se demostró que el tamaño de la localidad y la ubicación del hogar dentro de una región juegan un papel muy importante a la hora de reflejar una mayor o menor disposición al ahorro. Lo anterior es, sin duda, una consecuencia de las condiciones climatológicas, geográficas, sociales y económicas que prevalecen en dichos entornos. Por ejemplo, una vez que se toma en consideración el tamaño de la localidad, se encontró que, a medida que las viviendas se localizan en áreas más urbanizadas, la proporción de hogares con disposición al ahorro tiende a ser mayor respecto de las áreas menos urbanizadas; aunque también en estos hogares es donde se consume más electricidad. Este resultado corrobora el cumplimiento de la paradoja de Jevons y refuerza la idea de estimular las conductas proecológicas.

Respecto a los determinantes del ahorro, se encontró que las características del hogar, el inventario de equipos y la compra de enseres eléctricos con cierta conciencia proecológica son variables importantes para estimular el cuidado de la electricidad. En tanto que el precio del servicio no presentó un efecto sobre dicha disposición. Por lo que, en el contexto mexicano, en donde el mercado eléctrico se encuentra regulado y subsidiado, una estrategia de precios para estimular la DA no es efectiva.

Vale la pena comentar que, debido a la naturaleza de la encuesta, la unidad de análisis es el hogar, pero sería altamente recomen- 
dable estudiar el tema de la disposición al ahorro a nivel individual. También sería conveniente llevar a cabo un experimento controlado y monitoreado a lo largo de un periodo de tiempo, así como estudiar a profundidad y de manera cualitativa a una muestra de hogares en cada región para identificar y describir de mejor forma sus patrones de uso.

\section{Bibliografía}

Abrahamse, W. y Steg, L. (2009). How do socio-demographic and psychological factors relate to households' direct and indirect energy use and savings? Journal of Economic Psychology, 30, 711-720. https://www.rug.nl/staff/e.m.steg/abrahamsestegenergy. pdf

Agostini, C., Plottier, C. y Saavedra, E. (2012). La demanda residencial de energía eléctrica en Chile. Economía Chilena, 15(3), 6483. https://si2.bcentral.cl/public/pdf/revista-economia/2012/dic/ recv15n3dic2012pp64-83.pdf

Ajzen, I. (1991). The theory of planned behavior. Organizational Behavior and Human Decision Processes, 50(2), 179-211. https://www.sciencedirect.com/science/article/abs/pii/07495978 9190020T

Athukorala, W., Wilson, C., Managi, S. y Karunarathna, M. (2019). Household demand for electricity: The role of market distortions and prices in competition policy. Energy Policy, 134, 110932. https://www.sciencedirect.com/science/article/abs/pii/S03014 21519305191

Bartiaux, F. y Gram-Hanssen, K. (2005). Socio-political factors influencing household electricity consumption: A comparison between Denmark and Belgium. European Council for an Energy Efficient Economy, Panel 6. Dynamics of consumption. https:// www.eceee.org/library/conference_proceedings/eceee_Summer Studies/2005c/Panel_6/6131bartiaux/

Börsch-Supan, A. (1990). On the compatibility of nested logit models with utility maximization. Journal of Econometrics, 43(3), 373- 
388. https://www.sciencedirect.com/science/article/abs/pii/0304 40769090126E

Boudet H., Flora, J. y Armel, K. (2016). Clustering household energy-saving behaviours by behavioural attribute. Energy Policy, 92, 444-454. https://peec.stanford.edu/sites/g/files/sbiybj9616/f/ clustering_energy_behaviors.pdf

Brounen, D., Kok, N. y Quigley, J. (2012). Residential energy use and conservation: Economics and demographics. European Economic Review, 56(5), 931-945. https://www.sciencedirect.com/ science/article/pii/S0014292112000256

Cone, J. y Hayes, S. (1985). Environmental problems. Behavioral solutions. Nueva York, NY: Cambridge University Press.

Corral, V. (2006). Contribuciones del análisis de la conducta a la investigación del comportamiento pro-ecológico. Revista Mexicana de Análisis de la Conducta, 32, 111-127. http://journals. iztacala.unam.mx/index.php/smac/article/viewFile/51/53

Corral, V. (2010). Psicología de la sustentabilidad: un análisis de lo que nos hace pro ecológicos y pro sociales. México: Trillas.

Cramer, J., Miller, N., Craig, P., Hackett, B., Dietz, T., Vine, E., Levine, M. y Kowalczyk, D. (1985). Social and engineering determinants and their equity implications in residential electricity use. Energy, 10(12), 1283-1291. https://www.sciencedirect.com/ science/article/abs/pii/0360544285901392

Dietz, T., Gardner, G., Gilligan, J., Stern, P. y Vandenbergh, M. (2009). Household actions can provide a behavioral wedge to rapidly reduce U.S. carbon emissions. Proceedings of the National Academy of Sciences, 106(44), 18452-18456. https://www.pnas. org/content/106/44/18452

Ek, K. y Söderholm, P. (2010). The devil is in the details: Household electricity saving behavior and the role of information. Energy Policy, 38(3), 1578-1587. https://www.sciencedirect.com/science/ article/abs/pii/S0301421509008908

Guo, Z., Zhou, K., Zhang, C., Lu, X., Chen, W. y Yang, S. (2018). Residential electricity consumption behavior: Influencing factors, related theories and intervention strategies. Renewable and Sustainable Energy Reviews, 81(1), 399-412. https://www.science direct.com/science/article/abs/pii/S1364032117311164 
Henry, M. L., Ferraro, P. J. y Kontoleon, A. (2019). The behavioural effect of electronic home energy reports: Evidence from a randomised field trial in the United States. Energy Policy, 132, 12561261. https://www.sciencedirect.com/science/article/abs/pii/S03 01421519304094

Houthakker, H. (1951). Some calculations on electricity consumption in Great Britain. Journal of the Royal Statistical Society, 114, 359-371. https://rss.onlinelibrary.wiley.com/doi/epdf/ $10.2307 / 2980781$

Inglehart, R. (1997). Modernization and postmodernization: Cultural, economic, and political change in 43 societies. Princeton, NJ: Princeton University Press.

INEGI (Instituto Nacional de Estadística y Geografía) (2018a). Módulo de Hogares y Medio Ambiente 2017. México: INEGI. https:// www.inegi.org.mx/programas/mohoma/2017/

INEGI (Instituto Nacional de Estadística y Geografía) (2018b). Encuesta Nacional de los Hogares 2017. México: INEGI. https:// www.inegi.org.mx/programas/enh/2017/

Jevons, W. S. (1865). The coal question; an inquiry concerning the progress of the nation, and the probable exhaustion of our coal mines. Reino Unido: Macmillan Publishers.

Karlin, B., Davis, N., Sanguinetti, A., Gamble, K., Kirkby, D. y Stokols, D. (2014). Dimensions of conservation: Exploring differences among energy behaviors. Environment and Behavior, 46(4), 423-452. https://journals.sagepub.com/doi/10.1177/ 0013916512467532

Laicāne, I., Blumberga, A., Rošā, M. y Blumberga. D. (2014). Determinants of household electricity consumption savings: A Latvian case study. Agronomy Research, 12(2), 527-542. https://agronomy. emu.ee/wp-content/uploads/2014/05/2014_2_24_b5.pdf

Larios, A. (2014). La energía renovable en México: perspectivas desde el Balance Nacional de Energía 2012. Economía Informa, 385, 9099. http://www.economia.unam.mx/assets/pdfs/econinfo/385/07 lariosvazquez.pdf

Leahy, E. y Lyons, S. (2010). Energy use and appliance ownership in Ireland. Energy Policy, 38(8), 4265-4279. https://www.science direct.com/science/article/abs/pii/S0301421510002193 
Lezama, J. L. (2014). Política energética y sustentabilidad. La estrategia mexicana de ahorro y eficiencia de energía eléctrica en los hogares y la experiencia internacional. Ciudad de México: El Colegio de México, A.C.

Morales, D. y Luyando, J. (2016). Actitudes y conocimientos en el consumo de electricidad domiciliaria. Ciencia UANL, 77, 4650. http://cienciauanl.uanl.mx/wp-content/uploads/2016/03/art.DDE-LA-CFE.pdf

Mcloughlin, F., Duffy, A. y Conlon, M. (2012). Characterising domestic electricity consumption patterns by dwelling and occupant socio-economic variables: An Irish case study. Energy Build, 48(19), 240-248. https://arrow.tudublin.ie/cgi/viewcontent.cgi? article $=1056 \&$ context $=$ dubenart

Muljono, W. (2016). Electricity energy saving in Indonesia households: A look at communication campaign programs and the importance of environmental attitudes to help address the electricity crisis. Environmental Management and Sustainable Development, 5(2), 204-228. http://www.macrothink.org/journal/ index.php/emsd/article/view/9817

Ouyang, J. y Hokao, K. (2009). Energy-saving potential by improving occupants' behavior in urban residential sector in Hangzhou City, China. Energy and Buildings, 41(7), 711-720. https://www. sciencedirect.com/science/article/abs/pii/S0378778809000267

Özkan, H. A. (2016). Appliance based control for home power management systems. Energy, 114, 693-707. https:/www.infona.pl/ resource/bwmeta1.element.elsevier-15f84557-ea31-3806-84d5d3328263a17c

Poznaka, L., Laicane, I., Blumberga, D., Blumberga, A. y Rosa, M. (2015). Analysis of electricity user behavior: Case study based on results from extended household survey. Energy Procedia, 72, 79-86. https://www.sciencedirect.com/science/article/pii/ S187661021500702X

Saidur, R., Masjuki, H. H. y Jamaluddin, M. Y. (2007). An application of energy and exergy analysis in residential sector of Malaysia. Energy Policy, 35(2), 1050-1063. https://www.sciencedirect. com/science/article/abs/pii/S0301421506000930 
Sardianou, E. (2007). Estimating energy conservation patterns of Greek households. Energy Policy, 35(7), 3778-3791. https://www. sciencedirect.com/science/article/abs/pii/S0301421507000262 Sener (Secretaría de Energía). (2018). Prospectiva del Sector Eléctrico 2018-2032. Ciudad de México: Sener. https://base.energia. gob.mx/Prospectivas18-32/PSE_18_32_F.pdf

Shrum, L., McCarty, J. y Lowrey, T. (1995). Buyer characteristics of the green consumer and their implications for advertising strategy. Journal of Advertising, 24(2), 71-82. https://www.jstor.org/ stable/4188973?seq=1

Stern, P. (2000). New environmental theories: Toward a coherent theory of environmentally significant behavior. Journal of Social Issues, 56(3), 407-424. https://spssi.onlinelibrary.wiley.com/doi/ abs/10.1111/0022-4537.00175

Van den Broek, K. L., Walker, I. y Klöckner, C. (2019). Drivers of energy saving behaviour: The relative influence of intentional, normative, situational and habitual processes. Energy Policy, 132, 811-819. https://www.sciencedirect.com/science/article/ abs/pii/S0301421519304124

Van Raaij, W. F. y Verhallen, T. M. (1983). A behavioral model of residential energy use. Journal of Economic Psychology, 3(1), 39-63.https://www.cbsm.com/articles/31777-a-behavioral-modelof-residential-energy-use

Vergés, J. (2009). Mercado eléctrico, regulación y alternativas tecnológicas. Business Economy and Governments. Barcelona, España. http://webs2002.uab.es/jverges/Assaig\%20pdf/Mercado $\% 20$ eléctrico, \%20regulación $\% 20 \mathrm{y} \% 20$ alternativas\%20tec.pdf

Vicéns, J. (2008). La demanda de electricidad en España: importancia del sector residencial y estrategias de gestión activa. En Energía. Una visión económica (pp. 299-328). Madrid: Club Español de la Energía / Instituto Español de la Energía.

Wang, Z., Zhang, B., Yin, J. y Zhang, Y. (2011). Determinants and policy implications for household electricity-saving behavior: Evidence from Beijing, China. Energy Policy, 39(6), 3550-3557. https://doi.org/10.1016/j.enpol.2011.03.055

Yohanis Y. G., Mondol, J. D., Wright, A. y Norton, B. (2008). Real-life energy use in the UK: How occupancy and dwelling characteristics 
affect domestic electricity use. Energy and Buildings, 40(6), 10531059. https://www.sciencedirect.com/science/article/abs/pii/S03 7877880700223X?via\%3Dihub

Zhang, Y., Wang, Z. y Zhou, G. (2014). Determinants of employee electricity saving: The role of social benefits, personal benefits and organizational electricity saving climate. Journal of Cleaner Production, 66(1), 280-287. https:/www.sciencedirect.com/ science/article/pii/S0959652613006975

\section{Acerca de los autores}

Dionicio Morales Ramírez es maestro en Economía Industrial y doctor en Ciencias Sociales con orientación en Desarrollo Sustentable por la Universidad Autónoma de Nuevo León. Actualmente se desempeña como profesor-investigador en la Facultad de Ingeniería “Arturo Narro Siller" de la Universidad Autónoma de Tamaulipas. Pertenece al Sistema Nacional de Investigadores, nivel I. Su línea de investigación actual es: desarrollo socioeconómico, medio ambiente y sustentabilidad. ORCID: https://orcid.org/0000-0002-6859-6547

Algunas de sus publicaciones son:

Morales, D., Sánchez, N. y Martínez, V. (2020). El estilo de vida y su impacto sobre el estado nutricional en mujeres mexicanas: una aplicación del cuestionario Fantastic. Estudios Sociales, Revista de Alimentación Contemporánea y Desarrollo Regional, 30(55), 1-25. https://www.ciad.mx/estudiosociales/index.php/es/article/ view/835/527

Morales, D. Alvarado, E., Picazzo, E. y Tobías, J. (2018). Local perceptions of hydrocarbon exploitation operations in the Northeastern region of Mexico. Frontera Norte, 30(60), 111-130. https://fronteranorte.colef.mx/index.php/fronteranorte/article/ view/1139

Alvarado, E., Ortiz, J. y Morales, D. (2018). Factores que influyen en el emprendimiento de un micronegocio familiar en México. Revista de Economía del Rosario, 21(1), 153-180. https://revistas. urosario.edu.co/index.php/economia/article/view/6816 
Elías Alvarado Lagunas es doctor en Ciencias Sociales por la Universidad Autónoma de Nuevo León, institución donde actualmente es profesor e investigador en la Facultad de Contaduría Pública y Administración. Es miembro del Sistema Nacional de Investigadores, nivel I. Su línea de investigación actual es: desarrollo empresarial con énfasis en la sustentabilidad. ORCID: https://orcid.org/00000002-2751-7718

Sus más recientes publicaciones son:

Alvarado, E., Morales, D. y Ortiz, J. (2020). Determinantes de la probabilidad de robo a micronegocios en el área metropolitana de Monterrey. Revista de Economía, 37(94), 1-25. https://www. revista.economia.uady.mx/index.php/reveco/article/view/129 Alvarado, E., Valencia, K. y O. Iturralde. (2019). Impacto del entorno social en el robo a microempresas del área metropolitana de Monterrey. Economía, Sociedad y Territorio, 19(59), 1035-1058. http://www.scielo.org.mx/scielo.php?script=sci_abstract\&pid= S1405-84212019000101035\&lng=en\&nrm=iso\&tlng=es

Morales, D., Alvarado, E., Picazzo, E. y R. Tobías. (2018). Local perceptions of hydrocarbon exploitation operations in the Northeastern region of Mexico. Frontera Norte, 30(60), 111-130. http:// www.scielo.org.mx/pdf/fn/v30n60/0187-7372-fn-30-60-00111. pdf

Luis Jonás González Del Ángel es maestro en Construcción y doctor en Medio Ambiente con orientación en Desarrollo Sustentable por la Universidad Autónoma de Tamaulipas. Actualmente se desempeña como profesor-investigador en la Facultad de Ingeniería "Arturo Narro Siller" de la Universidad Autónoma de Tamaulipas. Pertenece al Cuerpo Académico de Economía, Empresa y Medio Ambiente. Su línea de investigación actual es: desarrollo socioeconómico, empresarial y medioambiente. ORCID: https://orcid.org/0000-0002-1773$580 \mathrm{X}$

Algunas de sus publicaciones son:

Gonzalez , L. (2019). Análisis del impacto mecánico de la adición de materiales reciclables en el concreto hidráulico y su desarrollo evolutivo. En M. D. Gracia, R. Tobías y R. Pichardo (coords.), 
Ingeniería, ciencia, tecnología e innovación en la cadena de conocimiento (pp. 155-169). Tampico, Tam., Mexico: Colofón.

Castan, J., Ibarra, S., Laria, J., Treviño, M., Pérez, J., González, L. y Castan, E. (2017). Intelligent ecosystems to increase urban traffic quality. Lecture Notes in Engineering and Computer Science: Proceedings of the World Congress on Engineering. Londres. http://www.iaeng.org/publication/WCE2017/WCE2017_pp221225.pdf

Recepción: 17 de octubre de 2019. Aceptación: 16 de abril de 2020. 
